\title{
BMJ Open Regional differences in endoscopic sinus surgery in Finland: a nationwide register-based study
}

\author{
Sanna Toppila-Salmi, ${ }^{1,2}$ Heikki Rihkanen, ${ }^{3}$ Martti Arffman, ${ }^{4}$ Kristiina Manderbacka, ${ }^{4}$ \\ IImo Keskimaki, ${ }^{4}$ Maija L Hytönen ${ }^{3}$
}

To cite: Toppila-Salmi S, Rihkanen $\mathrm{H}$, Arffman M, et al. Regional differences in endoscopic sinus surgery in Finland: a nationwide registerbased study. BMJ Open 2018;8:e022173. doi:10.1136/ bmjopen-2018-022173

- Prepublication history for this paper is available online. To view these files please visit the journal online (http://dx.doi org/10.1136/bmjopen-2018022173).

Received 20 February 2018 Revised 26 June 2018 Accepted 8 August 2018

Check for updates

(C) Author(s) (or their employer(s)) 2018. Re-use permitted under CC BY-NC. No commercial re-use. See rights and permissions. Published by BMJ.

${ }^{1}$ Haartman Institute, University of Helsinki, Helsinki, Finland

${ }^{2}$ Skin and Allergy Hospital,

Helsinki University Hospital and University of Helsinki, Helsinki,

Finland

${ }^{3}$ Department of

Otorhinolaryngology, Helsinki

University Hospital and

University of Helsinki, Helsinki,

Finland

${ }^{4}$ Health and Social Systems

Research Unit, National Institute for Health and Welfare, Helsinki, Finland

Correspondence to Dr Sanna Toppila-Salmi; Sanna.salmi@helsinki.fi

\section{ABSTRACT}

Objectives Endoscopic sinus surgery (ESS) is a common operation typically performed due to chronic rhinosinusitis (CRS). There are limited data on the nationwide ESS rate and factors contributing to its regional variation. The aim was to evaluate factors causing variation of ESS rate. Design Cross-sectional nationwide observational study. Setting A II patients undergoing ESS in Finland 20132015.

Population Nationwide Finnish population aged 15 years or over.

Main outcome measures ESS rate per 1000 inhabitants between 2013 and 2015 in all 21 hospital districts and independent factors for multilevel model analyses.

Methods We used the Finnish register data of all patients with CRS who underwent ESS in 2013-2015. Patients aged under 15 years and those with ESS due to neoplasia were excluded. The age and gender standardised ESS rates were calculated, and multilevel Poisson regression models were used to evaluate variation in ESS in the 21 hospital districts. The likelihood ratio test was applied to assess the statistical significance of random components in the models.

Results The nationwide annual rate of ESS is 0.71 per 1000 people in Finland. Hospital district rates varied from $0.25 / 1000$ (95\% Cl 0.18 to 0.32$)$ to $1.15 / 1000$ ( $95 \% \mathrm{Cl} 1.09$ 1.21). Compared with males, females undergo ESS significantly more frequently $(57 \%$ of the procedures), more often due to CRS without nasal polyps, and at a younger age (mean age 44.2 and 46.2 years, correspondingly). Multilevel analyses showed that lower age (between 24 years and 45 years) and availability/ease of medical services were independently associated with higher ESS rates.

Conclusions This study confirms marked regional variation in the ESS rate in Finland, explained only in part by patients' age and differing availability of medical services. To analyse ESS across different CRS phenotypes or to compare quality registers on ESS properly, more research on regional variation is needed.

\section{INTRODUCTION}

Chronic rhinosinusitis (CRS) is a clinical disorder encompassing a heterogeneous group of endotypes and two main phenotypes. ${ }^{1}$ It is one of the most common chronic adult health problems in the Western world

\section{Strengths and limitations of this study}

- Strengths of this study comprise a population-based nationwide design including all patients undergoing ESS in both public and private hospitals in Finland for the study years and information concerning the diagnoses, procedures performed and individual level demographic data.

- The completeness and accuracy of the hospital discharge register have consistently been shown to be good.

- Multilevel models enabled us to capture the hierarchical nature of the data (patients nested in hospital districts) allowing us to analyse the association more efficiently.

- A limitation is that no data on the need for sinus surgery are provided as no register data exist concerning the prevalence of conditions leading to endoscopic sinus surgery.

- Our data did not include information on health behaviours, earlier treatment of the condition or clinical information.

with a prevalence of $6 \%-11 \% \cdot{ }^{1-4}$ CRS has an independent and severe impact on the quality of life similar to chronic diseases such as asthma, chronic obstructive pulmonary disease (COPD) and diabetes. ${ }^{1}$ In the USA, the CRS-related healthcare costs are estimated to be $\$ 6.9-\$ 9.9$ billion dollars per year. $^{5}$

The predisposing factors of CRS include genetic and airborne factors in addition to host immunity. ${ }^{167}$ CRS is associated with airway allergy, asthma and COPD. ${ }^{18}$ CRS with nasal polyps (CRSwNP) and CRS without nasal polyps (CRSsNP) are considered to be phenotypes of CRS, with presumably different aetiologies and pathomechanisms. ${ }^{1}$ CRSwNP affects between $1 \%$ and $4 \%$ of the general population. ${ }^{1}$

Endoscopic sinus surgery (ESS) has been the most common operative approach for patients with CRS among whom maximal medical treatments have failed. ${ }^{1}$ ESS is a 
common procedure, with an estimated 250000 cases annually in the USA. ${ }^{9-11}$ ESS also creates a considerable financial burden. One study estimated the total cost of ESS to the UK National Health Service to be in excess of $£ 30$ million per year. ${ }^{12}$

Three non-population-based studies within the USA have shown ESS rates to vary according to geographic area or ethnicity. An observational cohort study performed on the MarketScan Commercial Claim and Encounters database yielded a mean ESS rate of 0.94 per 1000 employed, working-aged people in the USA. ${ }^{9}$ Moreover, the study showed fourfold to fivefold differences between the states with the highest and lowest rates. Venkatraman et al investigated a $20 \%$ sample of Medicare beneficiaries aged 65-99 years. Their study showed an increase in ESS rates for the period 1998-2006. ${ }^{13}$ The third study based on the MarketScan Medicaid database in the USA reported overall sinus surgery rates per 1000 people in the Medicaid population to range from 0.36 to 0.40 in 2009-2013. ${ }^{13}$ The ESS rate was significantly lower for African-Americans than for persons of Hispanic origin.

The reasons behind the detected geographic variation in ESS rates are not fully understood. Geographical variation might reflect a number of factors, including both underutilisation and overutilisation of surgery in the treatment of CRS, a lack of clinically unambiguous applicable guidelines and a lack of evidence in well-constructed randomised controlled trials. ${ }^{12}$ To our knowledge, no previous exhaustive nationwide studies have been conducted on ESS rates and factors affecting the likelihood of the surgical procedure. This nationwide study aimed to examine the number and rates of ESS performed due to inflammatory sinonasal diseases across Finland and putative factors explaining the difference.

\section{MATERIALS AND METHODS \\ Data}

The data of all patients who had undergone ESS by opening the ostiomeatal complex (DMB20) and/or opening the ethmoid sinuses (DNB20) due to sinonasal inflammations in Finland in 2013-2015 were extracted from the hospital discharge register, which registers all ESS procedures in Finland and is maintained by the Institute for Health and Welfare. Patients aged under 15 years and those operated on due to neoplastic lesions were excluded. Patients with CRS under 15 years of age were not included due to potential differences in their disease aetiopathogenesis and treatment. ${ }^{1}$ Age was classified into 5-year age brackets. Diagnosis was determined as CRSwNPs if any of the main or secondary diagnosis codes were J33. ${ }^{1}$ Furthermore, if any of the main or secondary diagnosis codes was J32 or the main diagnosis code was J01, the diagnosis was determined as CRSsNPs. ${ }^{1}$ Recurrent acute rhinosinusitis without nasal polyps (NPs) were regarded as belonging to the CRSsNP group. The rest of the procedures were classified into the group 'Other diagnoses'. The collected data included information on the ESS procedure, code of diagnosis (ICD10), gender, age, service provider and hospital district in which the patient was living at the time of ESS.

The data concerning hospitals performing ESS was classified into hospital districts, which own the public hospitals. Finland is divided into 21 hospital districts. The public hospitals within the district are owned by the federations of municipalities and mainly serving residents of their catchment area. In Finland, public medical care covers the vast majority (92\% in 2014) of all surgical interventions in different medical fields. Patient's possibility to choose any public hospital in the country increased in the beginning 2014, yet most patients have used the closest public hospital in their home hospital district also after that (https://www.kkv.fi/2016/kkv-selvityksia-3-2016). Both public and private healthcare providers report all hospitalisations and surgical procedures to the same nationwide hospital discharge database.

National population data in hospital districts were tabulated by gender and age in 5-year age brackets from Statistics Finland's website (http://www.stat.fi/til/vaerak/ index_en.html) for the years 2013-2015. The number of working-aged physicians and ENT doctors was retrieved from the website of the Finnish Medical Association (https://www.laakariliitto.fi/tutkimus/laakarityovoima).

The institute granted the permission to use hospital discharge register data for the patient population as the competent register authority in charge of the register.

\section{Patient and public involvement}

This study used national register data and did not involve patients in the recruitment or conduct of the study.

\section{Statistical analyses}

The data were tabulated by hospital district according to patients' residence. To reduce random variability, all analyses were performed for the whole study period from 2013 to 2015, but results were presented as annual averages. Due to the small number of cases, the smallest district of Åland islands (24 000 inhabitants aged over 15 years) was included only in the total number of ESS but excluded from the multilevel models.

We calculated age-adjusted and gender-adjusted rates of ESS in hospital districts using a direct standardisation method with the population structure of Finland in 2013-2015 as the standard population. Two-level Poisson regression models adjusted for age group with hospital district as random level and logarithm of population at risk as an offset variable were conducted to model the regional variation in ESS. Next, factors indicating proportion of operations performed by private ENT service providers, proportions of CRSwNP and CRSsNP and proportion of operations performed outside the hospital district of patients' residence in each stratum, as well as the density of physicians in hospital districts were added to the models. Models were estimated separately for men and women, as descriptive analyses revealed differing ESS profiles between them. Multilevel models were applied to 


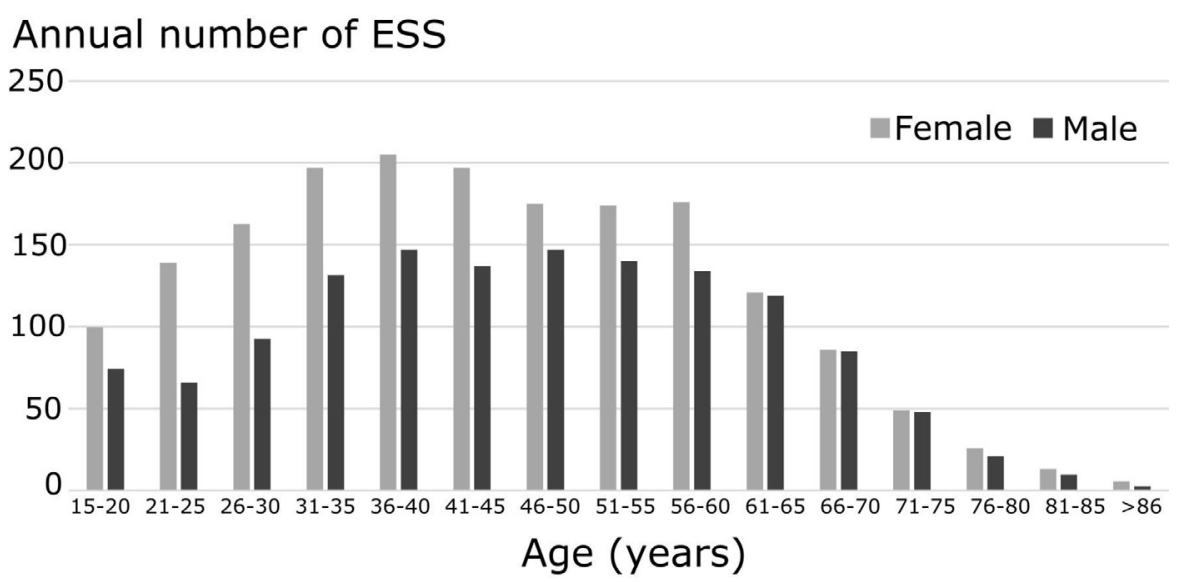

Figure 1 Age distribution of endoscopic sinus surgery (ESS) in Finland. Included are patients operated on during 2013-2015, presented as annual averages.

capture the hierarchical nature of the data, and effects of explaining factors were reported as risk ratios. Wald test was applied to assess the statistical significance of the variance term denoting variation at the hospital district level. Pearson's $\chi^{2}$ test was used to test the distribution of diagnoses in hospital districts. A 95\% CI was used to indicate statistical significance. Statistical analyses were performed by using SPSS V.22 and SAS V.9.3 software.

\section{RESULTS}

\section{Variation in ESS rates}

In 2013-2015, the total number of ESS was 9640, and the nationwide mean rate was 0.71 procedures per 1000 inhabitants. Three per cent of the patients underwent ESS twice or three times during this period. Operations were more common among women (age-standardised rates: $0.79 / 1000$ and $0.62 / 1000$, respectively). The mean age at the time of ESS was 44.0 (SD 15.9) years in females and 46.1 (SD 15.8) years in males (figure 1). The ESS rate of working-aged patients with CRS (aged 26-65 years) was higher $(0.86 / 1000)$ than the one among older patients with CRS aged 65 years or older $(0.35 / 1000)$. No significant differences were found in the age distribution of ESS patients between the hospital districts. Of all ESS procedures, $87 \%$ were performed by community-run public healthcare providers and $96 \%$ in the patient's home district (HD).

Seventy-two per cent of patients (62\% of males, $80 \%$ of females) who underwent ESS were diagnosed as having CRSsNP. Twenty per cent of patients (30\% of males, $13 \%$ of females) were diagnosed as having CRSwNP. Other diagnoses $(7 \%)$ included paranasal cysts or mucoceles, hypertrophy of turbinates and miscellaneous reasons.

The mean ESS rates per 1000 inhabitants aged over 15 years in the 21 districts are shown in the heatmap (figure 2). Hospital district rates varied from 0.25/1000 (95\% CI 0.18 to 0.32 ) to $1.15 / 1000$ (1.09 to 1.21 ) denoting a fourfold difference. The proportion of patients operated on for CRSsNP and CRSwNP varied significantly between districts $(\mathrm{p}<0.001)$.
Multilevel models of factors affecting the variation in ESS rate The multilevel models were created in order to study the level of variation in the probability of ESS between the hospital districts to examine whether certain factors had an independent effect on ESS and to assess whether the variation found between hospital districts could be explained by these factors.

Table 1 presents the results of multilevel modelling. In women, after adjusting for age, there was significant
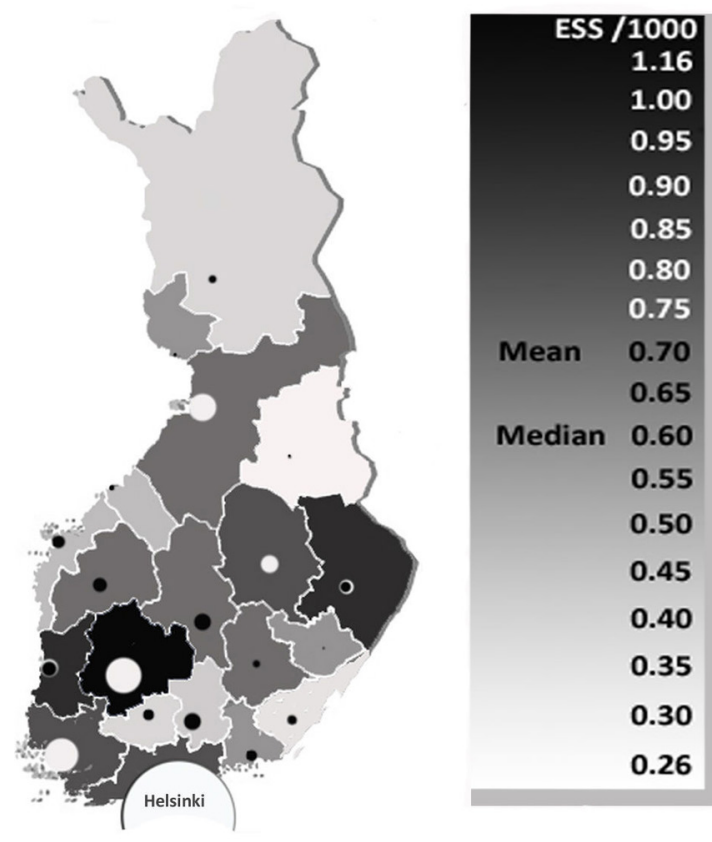

Figure 2 Age-standardised and gender-standardised rate of endoscopic sinus surgery (ESS) by hospital districts in Finland (mean of the years 2013-2015). Darker colours represent higher operation rates. The circle indicates the site of the central hospital, and the size represents the number of inhabitants within the district. Districts with white circles have a medical faculty. 
Table 1 Factors affecting ESS rates in men and women in an adjusted Poisson regression model

\begin{tabular}{|c|c|c|c|c|}
\hline \multirow[b]{3}{*}{ Variable } & \multicolumn{2}{|l|}{ Men } & \multicolumn{2}{|l|}{ Women } \\
\hline & Null model & Adjusted model & Null model & Adjusted model \\
\hline & RR $(95 \% \mathrm{Cl})$ & RR $(95 \%$ Cl) & RR $(95 \% \mathrm{Cl})$ & RR $(95 \% \mathrm{Cl})$ \\
\hline \multicolumn{5}{|l|}{ Age (years) } \\
\hline $15-24$ & 1.29 (1.11 to 1.51$)$ & 1.16 (0.96 to 1.39$)$ & 2.72 (2.39 to 3.11$)$ & 2.71 (2.31 to 3.18 ) \\
\hline $25-34$ & 1.79 (1.55 to 2.06 ) & 1.68 (1.45 to 1.95$)$ & 3.87 (3.41 to 4.39 ) & 3.87 (3.38 to 4.42$)$ \\
\hline $35-39$ & 2.59 (2.23 to 3.02 ) & 2.40 (2.05 to 2.81$)$ & 4.65 (4.06 to 5.32 ) & 4.63 (4.00 to 5.36$)$ \\
\hline $40-44$ & 2.57 (2.20 to 3.00 ) & 2.48 (2.12 to 2.90$)$ & 4.80 (4.19 to 5.50$)$ & 4.76 (4.13 to 5.49$)$ \\
\hline $45-49$ & 2.49 (2.14 to 2.90$)$ & 2.35 (2.01 to 2.75 ) & 3.77 (3.28 to 4.34$)$ & 3.73 (3.24 to 4.29$)$ \\
\hline $50-54$ & 2.40 (2.06 to 2.80$)$ & 2.24 (1.91 to 2.63 ) & 3.60 (3.13 to 4.14$)$ & 3.56 (3.09 to 4.10$)$ \\
\hline $55-59$ & 2.23 (1.90 to 2.60 ) & 2.06 (1.75 to 2.42 ) & 3.72 (3.24 to 4.27 ) & 3.69 (3.21 to 4.25$)$ \\
\hline $60-64$ & 1.99 (1.69 to 2.33 ) & 1.88 (1.59 to 2.23 ) & 2.48 (2.14 to 2.88 ) & 2.46 (2.11 to 2.86 ) \\
\hline $65-69$ & 1.74 (1.48 to 2.06 ) & 1.72 (1.46 to 2.04 ) & 1.92 (1.63 to 2.25 ) & 1.90 (1.62 to 2.23$)$ \\
\hline $70+$ & 1.00 & 1.00 & 1.00 & 1.00 \\
\hline$\%$ private ORL & & 2.24 (1.49 to 3.38 ) & & 1.22 (0.74 to 2.01$)$ \\
\hline$\%$ CRSsNP & & 0.73 (0.42 to 1.30$)$ & & 1.00 (0.56 to 1.76$)$ \\
\hline$\%$ CRSwNP & & 0.96 (0.56 to 1.66$)$ & & 1.27 (0.67 to 2.42 ) \\
\hline$\%$ outside HD & & 0.49 (0.30 to 0.81$)$ & & 0.89 (0.49 to 1.64$)$ \\
\hline Physician density & & 1.11 (0.99 to 1.25$)$ & & 1.16 (1.02 to 1.33$)$ \\
\hline HD (Var(SE)) & $0.1299(0.0464)$ & $0.0751(0.0287)$ & $0.1303(0.0463)$ & $0.1012(0.0381)$ \\
\hline
\end{tabular}

CRSsNP, chronic rhinosinusitis without nasal polyps; CRSwNP, chronic rhinosinusitis with nasal polyps; ESS, endoscopic sinus surgery; $\mathrm{HD}$, home district; ORL, otorhinolaryngologist operation; RR, risk ratio.

variation at the hospital district level $(\mathrm{p}<0.001)$. After adjusting for independent factors, the regional variation in ESS rates remained significant $(\mathrm{p}<0.001)$. In men, we found significant variation at the hospital district level after adjusting for age $(\mathrm{p}<0.001)$. Again, after adjustment for independent factors, the regional variation in ESS remained significant $(p<0.001)$. No differences from the national average were found in half of the home districts after controlling for age only among both men and women. Controlling for other independent factors levelled out the operation risk compared with the national average in seven home districts among men and four among women. However, part of the variation between hospital districts remained unexplained.

Of the independent factors, younger age $(p<0.001)$ and high density of physicians $(p=0.026)$ were significantly associated with a high ESS rate (table 1 ). Women aged 40-44 years were operated on 4.76 times more frequently than women aged over 70 years. Among men, age $(\mathrm{p}<0.001)$ and the proportion of ESS performed by private service providers $(\mathrm{p}=0.0001)$ were associated with high operation rates in the district, whereas ESS performed outside the patient's home district $(p=0.004)$ was significantly associated with a low rate.

The proportion of patients operated on due to CRSwNP varied from one district to another $(p<0.001)$. The range was from $10 \%$ to $33 \%$. In multivariate multilevel models, the diagnosis did not explain the risk on the operation rate.

\section{DISCUSSION}

To reduce inefficient or unwarranted practice patterns and to improve the overall performance of the health system, it is essential to define the variations in healthcare and the underlying reasons. ${ }^{914}$ We found regional variation in ESS not explained by variations in gender and age of the patient population.

\section{Comparison with other studies}

In Finland, the nationwide mean annual rate of ESS among adult patients was $0.71 / 1000$. The number is similar to what another study group has detected in the UK population. ${ }^{12}$ The ESS rate of working-aged people $(0.86 / 1000)$ in the current study is fairly close to that reported among the employed cohort in the USA $(0.94 / 1000) .{ }^{9}$ We demonstrate here that elderly people in Finland are operated on considerably less frequently $(0.35 / 1000)$ than their peers in the USA $(0.92 / 1000) .{ }^{13}$ In the present study, there was marked variability in ESS rates between the 21 hospital districts after standardisation of age and gender. We found a fourfold difference between the highest and the lowest regional operation rate. This is in line with the findings in the $\mathrm{USA}^{913}$ where up to fivefold differences between the states were observed. Private hospitals, in which $13 \%$ of all ESS procedures were performed, lack precise catchment areas in Finland and thereby population at risk. Yet, the risk ratios of the current study suggest that the proportion of private hospitals in the hospital district did have an effect on ESS 
operations. Nevertheless, the present nationwide study of Finland shows that regional differences exist also in a country with a universal nationwide healthcare system where the procedures are performed mostly in publicly financed institutions.

\section{The effect of independent factors to ESS variation}

We demonstrated that ESS is performed 1.3 times more frequently on women than on men. This is in accord with the expected population-based male-to-female ratio of CRS. CRS is estimated to be 1.1-2 times more common in women than in men. ${ }^{215}$ The infection rates in females aged between 20 and 34 years are higher probably due to a greater occupational risk to be exposed to infections relative to men. ${ }^{1617}$ This might, in part, enhance the development of CRS in females. In the present study, the mean age of operated females was lower than that of males. This might be due to differences in host-environment interactions and pathomechanisms. ${ }^{18}$ It is also possible that women use more general healthcare services. ${ }^{19}$

In this study, the ESS rate of working-aged people (26-65 years) was 2.5 times higher than that of retirement-aged people. Moreover, the multilevel models showed that the patient's age had an independent effect on ESS rate across Finland, which could putatively be related to differences in life style, operation eligibility or probability to have been already operated.

It is difficult to evaluate the population-based prevalence of CRSwNP and CRSsNP due to limitations of diagnostic methods in primary care. In the European population, the prevalence of CRSwNP has been estimated at 1\%-4\% and CRSsNP about $10 \% .{ }^{120}$ In the present study, $20 \%$ of ESS was performed on patients with CRSwNP. This is slightly higher than the ratio of CRSwNP and CRSsNP phenotypes in the literature cited above. It is consistent with the greater need for surgery with nasal polyposis.

ESS due to CRSwNP was performed nearly three times more frequently on males than on females. In a retrospective, cross-sectional American study, data on CRS patients and their non-CRS controls were analysed. ${ }^{18}$ The authors found that the proportion of females with CRSwNP was $38 \%$ and that these women had more severe disease than men. ${ }^{18}$ In our study, $27 \%$ of the operated CRSwNP patients were female. This figure is in line with the previous study. Hypertrophy or air cells of the turbinates does not normally fulfil criteria for ESS and could in part be related to wrong or incomplete insertion of diagnosis code.

\section{Potential factors behind variation in ESS}

The multilevel models showed that in females, young age and high density of physicians were significantly associated with a high ESS likelihood. In males, independent factors associated with a high probability of ESS were young age and a high proportion of ESS performed by private ENT specialists. ESS performed out of the patient's home district was associated with a low operation likelihood. Excluding the age factor, these findings reflect the impact of availability on operation rates. Availability of medical services would mean any kind of ease to get the medical service including distance, queuing time, easiness to get appointment and so on. Higher ESS rates were detected also in hospital districts with a high density of ENT specialists as well as in hospital districts having a university hospital with medical faculty. Yet, due to multicollinearity, these factors could not be included in the multilevel Poisson model. Taken together, our findings suggest that, in addition to the population's age structure, the availability of medical services has an independent effect on ESS probability.

\section{Putative other reasons for regional variation in ESS rate}

In both females and males, after adjusting for age and factors related to the availability of medical services, a clear regional variation remained in ESS, probably due to in part relative indications for ESS In industrialised countries, ESS rate might depend on informed patient choice and the treatment strategies available and the rate can vary extensively because of differences in professional opinions. ${ }^{14}{ }^{21}$ Future research should focus more on evidence-based treatments for CRS and indications of ESS in different disease course and phenotypes.

\section{Strengths and limitations of the study}

Strengths of this study comprise a population-based nationwide design and reliable register-based data, including information on all surgical procedures performed in Finland. ${ }^{22}$ Meticulously collected data on diagnosis, operating hospital and patient's municipality of residence as well as data on the density of physicians and ENT specialists are readily available.

The study includes some limitations that must also be addressed. No data on the need for sinus surgery are provided, as we examined the numbers of operated patients instead of the CRS patient population as no register data exist on the total patient population eligible for the operation. Furthermore, our data did not include information on patient's socioeconomic status, general health, subjective nasal symptoms or X-ray findings. Despite relatively small regional variation in socioeconomic or ethnic status of the Finnish population, some regional dissimilarities might exist, which could affect the probability of being operated on. We acknowledge that the register-based data have limited information on background factors and thus they could not be added to the multilevel models. A limitation is that paediatric patients with CRS under 15 years of age were not included due to potential differences in their disease aetiopathogenesis and treatment. Future studies should address potential variation in ESS in paediatric population. We acknowledge that lack of data of patient's allergy and other associating conditions, as well as of ESS costs limit interpretation of the results.

\section{CONCLUSIONS}

This nationwide study shows that high regional variation in ESS rate exists in Finland where the vast majority of 
procedures are performed in a public healthcare system. Patient's age and the availability of medical services are associated with ESS probability. However, these factors explained ESS rate variability only in part. This might create a problem on an unbiased quality control among operating institutions.

Contributors All authors participated in the planning and conception of the study and the analytical strategy. HR, MA, KM and IK performed the data management, analyses, tables and figures. HR, SKT-S and MH wrote the manuscript. All authors critically reviewed the manuscript.

Funding The study was supported in part by a research grant from the Aatos Erkko Foundation. All authors declare the statement of independence of researchers from the funder.

Competing interests None declared.

Patient consent Not required.

Ethics approval Ethical consent was obtained from the Research Ethics Committee of the Finnish National Institute for Health and Welfare (approval number 9/2016/\$746).

Provenance and peer review Not commissioned; externally peer reviewed.

Data sharing statement № additional data available.

Open access This is an open access article distributed in accordance with the Creative Commons Attribution Non Commercial (CC BY-NC 4.0) license, which permits others to distribute, remix, adapt, build upon this work non-commercially, and license their derivative works on different terms, provided the original work is properly cited, appropriate credit is given, any changes made indicated, and the use is non-commercial. See: http://creativecommons.org/licenses/by-nc/4.0/.

\section{REFERENCES}

1. Fokkens WJ, Lund VJ, Mullol J, et al. European position paper on rhinosinusitis and nasal polyps 2012. Rhinol Suppl 2012;23:3.

2. Hastan D, Fokkens WJ, Bachert C, et al. Chronic rhinosinusitis in Europe--an underestimated disease. A GA2LEN study. Allergy 2011;66:1216-23.

3. Kim JH, Cho C, Lee EJ, et al. Prevalence and risk factors of chronic rhinosinusitis in South Korea according to diagnostic criteria. Rhinology 2016;54:329-35.
4. Pilan RR, Pinna FR, Bezerra TF, et al. Prevalence of chronic rhinosinusitis in Sao Paulo. Rhinology 2012;50:129-38.

5. Smith KA, Orlandi RR, Rudmik L. Cost of adult chronic rhinosinusitis: a systematic review. Laryngoscope 2015;125:1547-56.

6. Toppila-Salmi S, van Drunen CM, Fokkens WJ, et al. Molecular mechanisms of nasal epithelium in rhinitis and rhinosinusitis. Curr Allergy Asthma Rep 2015;15:495.

7. Bohman A, Juodakis J, Oscarsson M, et al. A family-based genomewide association study of chronic rhinosinusitis with nasal polyps implicates several genes in the disease pathogenesis. PLoS One 2017;12:e0185244.

8. Chien CY, Tai SY, Wang LF, et al. Chronic obstructive pulmonary disease predicts chronic rhinosinusitis without nasal polyps: A population-based study. Am J Rhinol Allergy 2015;29:e75-e80.

9. Rudmik L, Holy CE, Smith TL. Geographic variation of endoscopic sinus surgery in the United States. Laryngoscope 2015;125:1772-8.

10. Bhattacharyya N. Ambulatory sinus and nasal surgery in the United States: demographics and perioperative outcomes. Laryngoscope 2010;120:635-8.

11. Svider PF, Darlin S, Bobian M, et al. Evolving trends in sinus surgery: what is the impact of balloon sinus dilation? Laryngoscope 2018;128.

12. Philpott $C$, Hopkins $C$, Erskine $S$, et al. The burden of revision sinonasal surgery in the UK-data from the Chronic Rhinosinusitis Epidemiology Study (CRES): a cross-sectional study. BMJ Open 2015;5:e006680.

13. Venkatraman G, Likosky DS, Morrison D, et al. Small area variation in endoscopic sinus surgery rates among the medicare population. Arch Otolaryngol Head Neck Surg 2011;137:253-7.

14. Wennberg JE. Time to tackle unwarranted variations in practice. BMJ 2011;342:d1513.

15. Ference $\mathrm{EH}$, Tan BK, Hulse KE, et al. Commentary on gender differences in prevalence, treatment, and quality of life of patients with chronic rhinosinusitis. Allergy Rhinol 2015;6:82-8.

16. Bachert $\mathrm{C}$, Holtappels $\mathrm{G}$. Pathophysiology of chronic rhinosinusitis, pharmaceutical therapy options. GMS Curr Top Otorhinolaryngol Head Neck Surg 2015;14:Doc09.

17. Heikkinen T, Järvinen A. The common cold. Lancet 2003;361:51-9.

18. Stevens WW, Peters AT, Suh L, et al. A retrospective, cross-sectional study reveals that women with CRSwNP have more severe disease than men. Immun Inflamm Dis 2015;3:14-22.

19. Kazanjian A, Morettin D, Cho R. Health care utilization by Canadian women. BMC Womens Health 2004;4(Suppl 1):S33-6874.

20. Chaaban MR, Walsh EM, Woodworth BA. Epidemiology and differential diagnosis of nasal polyps. Am J Rhinol Allergy 2013;27:473-8

21. Wennberg JE, Fisher ES, Skinner JS. Geography and the debate over Medicare reform. Health Aff 2002;Suppl Web Exclusives:W96-114.

22. Sund R. Quality of the Finnish hospital discharge register: a systematic review. Scand J Public Health 2012;40:505-15. 